\title{
AN ASSESSMENT OF THE ACCURACY OF THE DEEP SPACE NETWORK EXTRAGALACTIC REFERENCE FRAME
}

\author{
C.S. Jacobs and O.J. Sovers \\ Jet Propulsion Laboratory, \\ California Institute of Technology, \\ Pasadena, California, U.S.A. 91109
}

\begin{abstract}
The Deep Space Network (DSN) Radio Reference Frame consists of a catalog of angular positions for 281 extragalactic radio sources based on VLBI measurements made during the period from 1978 to 1992. A realistic assessment of the accuracy of these source position estimates must consider both modeled stochastic errors and systematic model deficiencies. Modeled stochastic errors include thermal noise (signal-to-noise ratio) and fluctuations in tropospheric refractivity due to the changing distribution of water vapor. These modeled errors result in a median formal position uncertainty of $\approx 0.3$ milliarcseconds (mas). In particular, we examine the effect of changing the model for inter-observation correlations of water vapor fluctuations on estimated parameters. Next, a comparison of our radio source positions with independently determined positions is presented as evidence of systematic errors at $\leq 0.5$ mas. We discuss several aspects of VLBI model accuracy focussing on tidal effects, antenna thermal expansion, pressure loading, source structure, precession and nutation. Prospects for reducing these errors are also discussed. We conclude by combining these estimates of modeled stochastic errors and systematic errors into an overall assessment of $<1.0$ mas for the current accuracy of the DSN extragalactic radio frame.
\end{abstract}

\section{INTRODUCTION}

A radio reference frame has been constructed, for the use by the Jet Propulsion Laboratory's (JPL) Deep Space Network (DSN), by measuring the angular coordinates of 281 extragalactic radio sources. Extragalactic objects were chosen for the construction of the radio reference frame because their extreme distance gives them high angular stability. In addition to the intrinsic scientific interest in reference frame stability, various applications require accurate and stable positions of the objects in the reference frame. These applications include deep space navigation (e.g. Border et al. 1982), earth orientation measurements (e.g. IERS 1992), and astrometry (e.g. Sovers et al. 1988, Treuhaft and Lowe 1991). The discussion will include a brief overview of our experimental program, a description of modeled stochastic errors, an assessment of the size of systematic model deficiencies that degrade the accuracy of the reference frame, and prospects for reducing these deficiencies. We conclude with our estimate of the realistic accuracy of the source coordinates which comprise the radio reference frame.

\section{RADIO REFERENCE FRAME EXPERIMENTS}

Our experimental program has been motivated by the need to provide a reference frame for navigating interplanetary spacecraft. The frame is defined by a catalog of radio source angular coordinates. Our observations are made using the very long baseline interferometric (VLBI) technique with the DSN 34m and 70m antennas near Goldstone, California, Madrid, Spain, and Tidbinbilla, Australia. These antennas are used in pairs to form two baselines: California to Madrid $(8,400 \mathrm{~km})$ and California to Tidbinbilla $(10,600 \mathrm{~km})$; no direct observations have been done between Madrid and Tidbinbilla. The data analyzed for this paper were taken over the period 1978 to 1992 and consist of 17300 observable delay and delay rate pairs. Data acquisition was done with the MkII $(1978-89, \approx 8000$ observations) and MkIII (1988-92, $\approx 9000$ observations) systems. Simultaneous 
S-band $(2.3 \mathrm{GHz})$ and X-band $(8.4 \mathrm{GHz})$ signals were recorded in order to calibrate charged particle effects. The most recent catalog based on the data described above is denoted JPL 7892-60. It contains right ascension and declination estimates for 281 sources spread fairly evenly over the sky from declination $-45^{\circ}$ to $+85^{\circ}$. The region within $10^{\circ}$ of the ecliptic plane has slightly denser coverage due to our need to provide sources for interplanetary navigation.

\section{MODELED STOCHASTIC ERRORS}

The first step in assessing the accuracy of the DSN radio reference frame is to account for known stochastic errors. The two principal sources of known stochastic error are thermal noise in the receiving system and the noise caused by fluctuations in tropospheric refractivity. The thermal noise is a function of the sensitivity of the receiving system. A typical DSN experiment uses two $34 \mathrm{~m}$ diameter antennas with aperture efficiencies of $\approx 70 \%$; system temperatures of $\approx 35^{\circ} \mathrm{K}$ and a recorded bandwidth of $28 \mathrm{MHz}$ (or $56 \mathrm{Mbit} / \mathrm{sec}$ with Nyquist sampling). Typical sources have a flux density of somewhat less than 1 Jansky $\left(1 \mathrm{watt} / \mathrm{m}^{2} \mathrm{~Hz}\right)$. For this system the average thermal noise error is equivalent to $8 \mathrm{~mm}$ uncertainty in signal path delay or $\approx 0.2$ mas on a $10,000 \mathrm{~km}$ baseline.

The other modeled source of stochastic error is the observable noise caused by fluctuations in tropospheric refractivity. There are two alternative models of tropospheric noise that we use in our analysis. The first model accounts for fluctuations in tropospheric refractivity by estimating a new parameter for the the troposphere delay at zenith once every 3 hours, thereby implicitly accounting for temporal correlations in refractivity fluctuations. For this model, zenith parameter estimates used an observation weight due to troposphere noise of $4 \mathrm{~mm} / \sin (E)$ where $E$ is the elevation angle above the horizon, the factor $1 / \sin (E)$ scales the noise in proportion to the path through the troposphere, and the $4 \mathrm{~mm}$ constant was determined from data scatter. The main advantage of this model is that it is simple to calculate. The main disadvantage is that it ignores spatial correlations and only crudely accounts for temporal correlations in fluctuations in tropospheric refractivity. The alternative Treuhaft and Lanyi (1987) model accounts for both spatial and temporal correlations at the cost of greatly increased computational complexity. Briefly, Treuhaft and Lanyi model the spatial fluctuations in tropospheric refractivity using Kolmogorov turbulence theory and account for temporal correlations by assuming that the spatial fluctuations are moved in a "frozen flow" by $\mathrm{a} \approx 10 \mathrm{~m} / \mathrm{sec}$ wind.

In order to assess the relative merits of these alternative models, we have analyzed a subset of our data (1988-92, $\approx 50$ separate days or "experiments" recorded with MkIII system) using both models of tropospheric noise. The results show that the Treuhaft-Lanyi model improved the accuracy of our results as evidenced by reduced experiment-to-experiment scatter in the estimated distance between antennas (baseline length). For the 10,600 km California to Tidbinbilla baseline, the Treuhaft-Lanyi model reduced weighted RMS (WRMS) scatter from $25.7 \mathrm{~mm}$ to $18.4 \mathrm{~mm}$ while simultaneously reducing chi square per degree of freedom, $\chi_{\nu}^{2}$, from 1.4 to 0.9 . For the $8,400 \mathrm{~km}$ California to Madrid baseline, the Treuhaft-Lanyi model reduced WRMS scatter from $14.4 \mathrm{~mm}$ to $10.1 \mathrm{~mm}$ while simultaneously reducing $\chi_{\nu}^{2}$ from 2.7 to 1.7 . The $\chi_{\nu}^{2}=0.9$ for the $10,600 \mathrm{~km}$ baseline indicates that the observed $18.4 \mathrm{~mm}$ scatter is consistent with modeled errors (thermal error + Treuhaft-Lanyi troposphere). However, the $\chi_{\nu}^{2}=1.7$ for the $8,400 \mathrm{~km}$ baseline indicates that modeled errors do not account for the observed scatter and suggests there is $\approx 8 \mathrm{~mm}$ of unmodeled error $(\approx 0.2$ mas on $8,400 \mathrm{~km}$ baseline).

In summary, we model the stochastic errors due to thermal noise and fluctuations in tropospheric refractivity (with the Treuhaft-Lanyi model). When these error models are applied to the experiments described in section II, one obtains median formal source position errors of $\approx 0.3$ mas in both declination, $\delta$, and right ascension, $\alpha$ (scaled by $\cos \delta$ to give arclength). 


\section{SYSTEMATIC ERRORS DUE TO MODEL DEFICIENCIES}

The model used in constructing the DSN radio reference frame attempts to accurately describe radio signal path delays at the $1-\mathrm{cm}$ level or better. In this section we will give a summary of the model currently in use, present an external measure of source coordinate accuracy, and finally discuss several aspects of the model that limit the accuracy of these coordinates.

The model that we are considering follows the IERS standards (McCarthy 1989) except for use of the ocean loading model of Scherneck (1991), the short period tidal UT1-UTC model of Wünsch and Busshoff (1992), and the tropospheric mapping function of Lanyi (1984). For a priori earth orientation we use the IERS-90C04 series (IERS 1992). More complete details of our VLBI model can be found in Sovers (1991). The model is adjusted by using least squares estimation techniques to solve for numerous parameters. The "global" parameters include the right ascension $(\alpha)$ and declination $(\delta)$ of all sources except $\alpha(3 \mathrm{C} 273)$ which is held fixed in order to provide the origin of right ascension. For each experiment (typically $24 \mathrm{hr}$ of data $=200-300$ observations), estimated parameters include one baseline vector, corrections to the celestial ephemeris pole (longitude and obliquity) relative to the IAU 1980 precession/nutation model (Seidelmann 1982, Wahr 1981) with the pole fixed to the IAU value on the reference day of May 20, 1983, piecewise linear clock parameters are estimated once every 2-24 hours (as required by the data), and a new zenith troposphere delay parameter every 12 hours. Observations are weighted by the combined covariance from thermal noise and Treuhaft-Lanyi troposphere fluctuations.

In order to obtain an external calibration of the accuracy of our radio reference frame we have compared JPL source coordinates to a recent Goddard Space Flight Center (GSFC) catalog of coordinates, based on data available to the Crustal Dynamics Project (CDP), and labeled G831. This comparison is summarized in Table 1. The GSFC catalog was selected for comparison to the JPL catalog because it has similar sky coverage and quality and because it is based on independent data and independent analysis software (Ma et al. 1990). Before differencing coordinates, a three dimensional rotation is determined (with an uncertainty of $\approx 0.2$ mas) and removed in order to place the catalogs into best coincidence by eliminating differences in JPL and GSFC frame orientation conventions, such as origin of right ascension and reference day (i.e. day on which nominal IAU precession/nutation is used without adjustment) for nutation model adjustments. Assuming the error covariance matrices of the two catalogs to be independent, $\chi_{\nu}^{2}=4.4$ for the JPL, GSFC coordinate differences. This indicates that the modeled formal uncertainties underestimate the true accuracy of the JPL and GSFC coordinates. In order to make $\chi_{\nu}^{2}=1$ we had to add 0.5 mas of white noise to each coordinate difference. This additional 0.5 mas serves as an upper bound for the level of unmodeled systematic error which is corrupting the JPL-GSFC catalog differences.

Table 1. Comparison of GSFC G831 and JPL 7892-60 Catalogs

\begin{tabular}{|c|c|}
\hline Rms uncertainty for 212 common sources & \\
GSFC G831: $\alpha \cos (\delta), \delta$ (mas) & $0.9, \quad 0.5$ \\
JPL 7892-60: $\alpha \cos (\delta), \delta$ (mas) & $0.8, \quad 0.6$ \\
Added noise ( $\alpha$ and $\delta$ ), mas & $0.5, \quad 0.5$ \\
\hline Rms difference (mas) : $\alpha \cos (\delta)$ & 1.0 \\
$\delta$ & 1.2 \\
\hline
\end{tabular}

In order to characterize the systematic errors which limit the accuracy of the JPL radio reference frame, we have carried out a number of internal consistency tests on the JPL data. These tests included examination of various components of our model such as tidal effects (ocean loading, ocean tide effect on earth rotation, solid earth tides), thermal expansion of antenna structures, atmospheric loading of the crust, source structure, precession and nutation. 
There are several components of our model (Sovers 1991) that are driven by the luni-solar tidal potential. These included ocean loading, ocean tidal effects on earth rotation, and solid earth tides. Recent work (e.g. Büllesfeld and Schuh 1986, Brosche et al. 1989) has indicated the need to improve these components of our model particularly in the semi-diurnal and diurnal frequency bands. Separately, each of these three effects may have model deficiencies at the $5 \mathrm{~mm}$ level $(\approx 0.1$ mas on 10,000 km baseline). Exploratory fits to the JPL data which added degrees of freedom at 4 nearly semi-diurnal $\left(K_{2}, S_{2}, M_{2}, N_{2}\right)$ and 4 nearly diurnal $\left(K_{1}, P_{1}, O_{1}, Q_{1}\right)$ tidal frequencies produced $8 \mathrm{~mm}$ improvement ( $>99 \%$ confidence level) in the RMS scatter of observed delays. Fits of this type (e.g. Herring and Dong 1991, Sovers, Jacobs and Gross 1992) are beginning to yield improved tidal models and thereby reduce tidal systematic errors. In addition to deficiencies in tidal models, we expect that the current model has deficiencies of $\approx 5 \mathrm{~mm}$ from failing to account for thermal expansion of the antenna structures. Variations in the antenna location due to seasonal variations in the atmosphere's load on the earth's crust are also unaccounted for at present and may be as large as $7 \mathrm{~mm}$ peak to peak. We are currently testing models for both thermal expansion and pressure loading (Rabbel and Schuh 1986) in an effort to reduce these sources of systematic error.

The extragalactic radio sources used to construct the JPL reference frame are not pointlike at the sub-milliarcsecond level. As a result, as the relative orientation of the baseline and source changes, the effective centroid of the source can vary at or above the formal source position uncertainties (e.g. Charlot 1990). Because these effects vary independently for each of our 281 sources on time scales as short as a few months, the effort required to model all the sources in the reference frame exceeds our resources. As an alternative to a complete structure model, we estimated apparent proper motions of the sources as linear time rates of change in right ascension, $\dot{\alpha}$, and declination, $\dot{\delta}$. Rates of the most frequently observed sources are uncertain by approximately $40 \mu \mathrm{as} / \mathrm{yr}$, indicating the limit of our sensitivity to apparent proper motions. Weighted RMS angular coordinate rates for 264 sources are 0.15 and $0.18 \mathrm{mas} / \mathrm{yr}$ for $\dot{\alpha}$ and $\dot{\delta}$, respectively. Given that the JPL and GSFC catalogs have differing mean epochs of observation for a given source, these $\dot{\alpha}$ and $\dot{\delta}$ may account for a significant portion of the 0.5 mas systematic error in the JPLGSFC comparison. We hope to examine this possibility in the near future. Overall a total of 13 $\dot{\alpha}$ exceed $3 \sigma_{\dot{\alpha}}$; only $9 \dot{\delta}$ were $>3 \sigma_{\dot{\delta}}$. Table 2 below shows the five most significant right ascension and declination rates, $\dot{\alpha}$ and $\dot{\delta}$, both in units of $\mu$ as/year. The number of observations per source, $N_{\text {obs }}$, time span of the observations in years, formal uncertainties in the rates, $\sigma_{\dot{\alpha}}$ and $\sigma_{\dot{\delta}}$, and rates normalized to the formal uncertainties, $\dot{\alpha} / \sigma_{\dot{\alpha}}$ and $\dot{\delta} / \sigma_{\dot{\delta}}$, are also given.

Table 2. Most Significant Right Ascension and Declination Rates ( $\mu \mathrm{as} / \mathrm{yr}$ )

\begin{tabular}{|l|c|c|c|c|c|}
\hline Source & $\begin{array}{c}\text { Time } \\
(\mathrm{yr})\end{array}$ & $N_{\text {obs }}$ & $\begin{array}{c}\text { Rate } \\
\mu \text { as } / \text { yr }\end{array}$ & $\begin{array}{c}\sigma \\
\mu \text { as } / \text { yr }\end{array}$ & $\sigma /$ rate \\
\hline$\dot{\alpha}$ 3C 273 & 13.3 & 241 & 276 & 40 & 6.9 \\
3C 345 & 12.2 & 348 & 252 & 47 & 5.4 \\
P 1123+26 & 12.2 & 213 & 211 & 43 & 4.9 \\
P 0528+134 & 12.2 & 179 & -219 & 45 & 4.9 \\
OX 036 & 4.0 & 62 & -649 & 140 & 4.7 \\
\hline & & & & & \\
$\delta$ OX 036 & 4.0 & 62 & 901 & 156 & 5.8 \\
P 0113-118 & 13.3 & 51 & 615 & 144 & 4.3 \\
P 0823+033 & 12.2 & 175 & 247 & 63 & 3.9 \\
P 2355-106 & 6.4 & 72 & 449 & 135 & 3.3 \\
3C 279 & 13.3 & 98 & 262 & 79 & 3.3 \\
\hline
\end{tabular}

The term apparent proper motion is used because changes in a source's brightness distribution (or structure) can shift the effective brightness centroid of a source and thus its measured position even if the source's core remains fixed. As a case in point, 3C 345 shows a $5.4 \sigma$ apparent proper 
motion of $\dot{\alpha}=252 \pm 47 \mu$ as/yr. However, Bartel et al. (1986) conclude that 3C 345's core shows no proper motion at the $20 \mu \mathrm{as} / \mathrm{yr}$ level while its surrounding jets are not as stable. The most significant rate inferred from our VLBI data is $\dot{\alpha}(3 C 273)$. This is another source known to have non-pointlike structure (e.g. Zensus and Pearson 1987). Thus these estimates of apparent proper motions may be more an indication of source structure changes than of true proper motion of the cores of the radio sources.

For applications which must extrapolate radio source coordinates to epochs far from the epochs of observation, uncertainty in the motion of the earth's celestial ephemeris pole (CEP) becomes a significant systematic error. The accuracy of the model of the CEP's motion is presently limited by the accuracy with which the precession constant and long-term (18.6-year period) nutation can be inferred from the data. Estimates from our data yield formal uncertainties of $0.4 \mathrm{mas} / \mathrm{yr}$ for precession, and 1.6 mas for the 18.6-yr nutation amplitude. Note that our VLBI data only cover 13.5 years of the 18.6-year nutation cycle, producing large correlations among these long-period parameters and leaving our estimates vulnerable to corruption by systematic mismodeling (which of course are not included in the formal uncertainties). Charlot et al. (1991) have taken advantage of the 23-year span of Lunar Laser Ranging (LLR) data to reduce these correlations in their combined VLBI-LLR analysis while simultaneously reducing the formal uncertainties several-fold. Thus we expect that analysis of combined data types or longer spans of VLBI data will reduce systematic uncertainty in models of the CEP's motion.

\section{CONCLUSIONS}

The JPL radio reference frame consists of a catalog of angular coordinates for 281 extragalactic sources. Our assessment of the accuracy of these coordinates includes both modeled stochastic errors and systematic model deficiencies. Modeled stochastic errors include thermal noise error and error due to fluctuations in tropospheric refractivity. These two error sources account for the $\approx 0.3$ mas median formal uncertainty of our angular coordinates. Our assessment of systematic errors included tidal effects, antenna thermal expansion, atmospheric pressure loading, source structure, precession and nutation. Individually, most of these model deficiencies are $\approx 0.1$ mas with source structure and precession/nutation being somewhat larger at $\approx 0.5$ mas. Within the next few years it appears feasible to produce models which will reduce these systematic effects. In particular, exploratory fits to VLBI data have estimated tidal, precession, and nutation parameters which significantly improve the fits. Models of antenna thermal expansion and pressure loading are currently being tested and are expected to reduce these sources of systematic error. We hope to partially account for source structure, one of the largest systematic errors, by modeling it as apparent proper motion. Considering all of these error sources gives a combined realistic accuracy estimate of $<1.0$ mas for the angular coordinates that comprise the JPL extragalactic radio reference frame.

ACKNOWLEDGEMENTS: R. P. Branson and R. F. Coker scheduled and analyzed many of the more recent DSN Mark III VLBI observing sessions. We appreciate access to the results of VLBI analyses by the Goddard Space Flight Center group. The work described in this paper was carried out by the Jet Propulsion Laboratory, California Institute of Technology, under contract with the National Aeronautics and Space Administration.

\section{REFERENCES:}

Bartel, N., T.A. Herring, M.I. Ratner, I.I. Shapiro, and B.E. Corey, "VLBI Limits on the Proper Motion of the Core of the Superluminal Quasar 3C 345", Nature, vol. 319, pp. 733-738, 1986.

J.S. Border, F.F. Donivan, S.G. Finley, C.E. Hildebrand, B. Moultrie, and L.J. Skjerve, AIAA Paper 82-1471, presented at the 1982 AIAA Conference, San Diego, Ca, August 1982.

Brosche, P., U. Seiler, J. Sündermann, and J. Wünsch, Periodic changes in Earth's rotation due to oceanic tides, Astron. Astrophys., 220, 318-320, 1989. 
Büllesfeld, F.-J. and H. Schuh, New harmonic development of the tide-generating potential ETMB 85 with applications on VLBI data analysis, Proc. X Internatl. Symp. on Earth Tides, ed. R. Vieira, 933-942, Madrid, 1986.

Charlot, P., Radio source structure in astrometric and geodetic very long baseline interferometry, Astron. J., 99, 1309-1326, 1990.

Charlot, P., O.J. Sovers, J.G. Williams, and X X Newhall, A global VLBI/LLR analysis for the determination of precession and nutation constants, Proc. of the 127th Colloquium of the IAU, Reference Systems , 228-233, U. S. Naval Observatory, Washington, D. C., 1991.

Herring, T.A. and D. Dong, "Current and Future Accuracy of Earth Rotation Measurements", Proceedings of the AGU Chapman Conference on Geodetic VLBI: Monitoring Global Change, 306-324, NOAA Tech. Rept. NOS 137 NGS 49, 1991.

International Earth Rotation Service, “1991 IERS Annual Report”, Observatory of Paris, 1992.

Lanyi, G.E., "Tropospheric Delay Effects in Radio Interferometry", NASA JPL TDA Prog. Rept. 42-78, pp. 152-159, Jet Propulsion Laboratory, Pasadena, California, April-June, 1984.

Ma, C., D.B. Schaffer, C. De Vegt, K.J. Johnston, and J.L. Russell, “A Radio Optical Reference Frame I. Precise Radio Source Positions Determined by Mark III VLBI: Observations from 1979 to 1988 and a Tie to the FK5", Astronomical Journal, vol. 99, pp. 1284-1298, 1990.

McCarthy, D.D., IERS Standards, IERS Tech. Note 3, International Earth Rotation Service, Paris Observatory, Paris, France, 1989.

Rabbel, W. and H. Schuh, The influence of atmospheric loading on VLBI experiments, J. Geophysics, 59, 164-170, 1986.

Scherneck, H.G., "A Parameterised Solid Earth Tide Model and Ocean Tide Loading Effects for Global Geodetic Baseline Measurements", Geophys. J. Int., vol. 106, pp. 677-694, 1991.

Seidelmann, P.K., "1980 IAU Nutation: The Final Report of the IAU Working Group on Nutation", Celestial Mechanics, vol. 27, pp. 79-106, 1982.

Sovers, O.J., C.D. Edwards, C.S. Jacobs, G.E. Lanyi, K.M. Liewer, and R.N. Treuhaft, "Astrometric Results of 1978-1985 Deep Space Network Interferometry: The JPL 1987-1 Extragalactic Source Catalog", Astronomical Journal, vol. 95, pp. 1647-1658, 1988.

Sovers, O.J., "Observation Model and Parameter Partials for the JPL VLBI Parameter Estimation Software "MODEST"-1991", Publication 83-39, Rev. 4, Jet Propulsion Laboratory, Pasadena, California, August 1, 1991.

Sovers, O.J., C.S. Jacobs, and R.S. Gross, "Measuring Rapid Ocean Tidal Earth Orientation Variations with VLBI", submitted to J. Geophys. Res., November, 1992.

Treuhaft, R.N. and G.E. Lanyi, "The Effect of the Dynamic Wet Troposphere on Radio Interferometric Measurements", Radio Science, vol. 22, pp. 251-265, 1987.

Treuhaft, R.N. and S.T. Lowe, “A Measurement of Planetary Relativistic Deflection", Astronomical Journal, vol. 102, pp. 1879-1888, 1991.

Wahr, J.M., "The Forced Nutations of an Elliptical, Rotating, Elastic, and Oceanless Earth", Geophys. J. Roy. Astron. Soc., vol. 64, pp. 705-727, 1981.

Wünsch, J. and J. Busshoff, "Improved Observations of Oceanic UT1", Research Note for Astronomy and Astrophysics, in press, accepted 10 July 1992.

Zensus, J.A. and T.J. Pearson, eds., "Superluminal Radio Sources", Cambridge Univ. Press, New York, New York, 1987. 